\title{
Agent-based Models as Fictive Instantiations of Ecological Processes
}

\author{
Steven L. Peck
}

\begin{abstract}
It has been argued that problems in computer simulation bear enough resemblance to recognized issues in the philosophy of modeling that they only pose philosophical challenges analogous to those found in standard analytic models used to represent the natural world. Agent-based models have become important for understanding the interactions among organisms in ecological and evolutionary systems. Like the complexity found in natural systems, these models allow emergent patterns to arise from lower-level processes. The use of these models presents several philosophical problems to understanding how a simulation represents and what role it can play in scientific discourse. One fruitful way to look at agent-based models is as instantiations: a representational mode that recreates a type of the system with which one is working. I explore whether agent-based models present new challenges for philosophy of science and why these types of models are relevant for understanding emergent systems. I argue that certain types of ecological systems may be examined more substantively with agential models. I also suggest that these models might be described as fictions and require deeper hermeneutic engagement than non-simulation models.
\end{abstract}

\section{KEYWORDS \\ Agent-based models $\bullet$ Ecological modeling $\bullet$ Instantiations \\ Models as fictions • Philosophy of simulation}

\section{Introduction}

Frigg and Reiss (2009) argue that philosophical problems in simulation bear enough resemblance to recognized issues in the philosophy of modeling that they only pose challenges analogous to those found in standard analytic models used to represent natural systems. They suggest that there are no new philosophical problems in computer simulation modeling beyond those found in traditional mathematical modeling. Winsberg (2009) has countered that there appear to be genuinely new epistemological problems in simulation modeling because the knowledge obtained from them is 'downward, motley, and autonomous.' Here I draw out some specific ways that these epistemological problems are manifest in complex ecological simulation, especially in agent-based models. These models contain novel features that were impossible to anticipate prior to the computer revolution, and continue to present difficulties and challenges of both a practical and philosophical nature (Humphreys 2002).

\footnotetext{
$\S$ Department of Biology, Brigham Young University, Provo, UT 84602 USA

E-mail: steven peck@byu.edu
}

Received 5 August 2011; Revised 2 March 2012; Accepted 6 March 2012 
In this paper I weigh in on some of these issues and elaborate why I think that agent-based ecological simulations are more complicated, and more interesting. I will focus on three things. First, I suggest that a fruitful way to look at agent-based models is as instantiations: a representational mode that recreates the type of the system with which one is working. Rather than a representation based upon mathematical or pictorial description, an instantiation recreates, in part, that same system with objects that have formal attributes similar to those found in the target system. Second, I explore whether agent-based models present new problems for philosophy of science and why these types of models will be relevant for understanding emergent systems. I argue that certain types of ecological systems may be examined more substantively with agential models (Odenbaugh 2005b; De Roos and Persson 2005). Third, I highlight that these models require a deeper hermeneutic engagement than analytic models. Current discussions within philosophy of science that fruitfully critique models as fictions offer a helpful perspective for better understanding these types of systems. These viewpoints may be useful for simulations of various types and across different disciplines, but my focus will be on agent-based ecological models. I show that these models pose many novel and challenging issues that require new conceptual work by philosophers of science.

\subsection{A few preliminaries on ecological theories}

Before we talk about agent-based simulation in ecology, there are some things about ecology to keep in mind. Ecological systems are arguably among the most complex systems found in nature. They often rival the complexity of such multidimensional and challenging sciences as developmental biology and neuroscience. The reasons for ecology's complexity are multifarious.

First, ecological systems are hierarchical, with higher-level processes constraining and influencing lowerlevels, and, in turn, lower-level processes contributing to the structure and function of higher-levels. These multiple scales of interaction can lead to complicated dynamics.

Second, ecological systems are numerically complex; they are comprised of a large number of players in networks of interacting organisms, which structure and are structured by many abiotic elements. Food webs, competitive interactions among and within species, structural dynamics of the physical environment produced by both abiotic and biotic influences, animal behavior and all this entails, are just a few pieces of the composite environmental milieu. For example, soils and plant community composition can be dramatically influenced by differences in solar energy shaped by the topology of landscape-level factors.

Third, ecological systems are historical in nature. They are contingent on accidents of colonization by differing organisms, accidents of weather and climate, perturbations caused by natural and human processes, and other influences that affect the historical development of an ecosystem. This contingency is the result of a complex evolutionary history in which species have evolved and coevolved over millions of years, creating interdependencies, reliances, mutualisms, and other symbiotic relationships that structure ecological communities.

Fourth, the part-whole relationships of ecosystem components are a fundamental aspect of these systems, even though in many ways they are contingent and accidental. The species present form relationships with other components, but do not have functional relationships to the 'whole' in the same way that the components of organisms do. The predator's regulation of prey does not serve as a function toward some ecosystem goal in the same way that a predator's heart functions toward pumping blood. This is because ecosystems as a whole are not evolved structures in the same sense that organisms are. However, ecosystems do have tendencies and capacities. For example, grassland or wetland ecosystems seem to function similarly in many parts of the world despite being composed of different species of plants, animals, and soil organisms. In short, ecosystems are complex spatially, temporally, and hierarchically. They also have numerous contingent features due to history and geography.

These features have fostered the argument that there may be no laws in ecology and that ceteris paribus conditions dominate, if not swamp, most aspects of ecological systems (Cooper 2003). Ecological theory has been hard to come by and, when proposed, tends to be ecosystem, species, or location specific. Many instances are so general or even cartoonish - like the 'law' of exponential growth - that they are hard to apply to actual organisms in real ecosystems except in broadest outline (Hall 1988). Ecology has proven to be a theory-poor science. Even recent attempts at providing unifying concepts seem limited and only 
marginally successful. This lack of generality, however, does not imply that all aspects of ecological theory are in vain. For example, in community ecology, widespread patterns have been noted that rely on fundamental processes that can be captured much like the theory of evolution by natural selection does, through pointing out how those processes (including natural selection) create specific patterns regardless of species composition (Roughgarden 2009; Vellend 2010; Grimm et al. 2005).

How can modeling, as conventionally understood, make any headway with such systems? Agent-based simulation seems to be one way (Colyvan et al. 2009; Caprio et al. 2008). The history of computer simulation in ecology has been controversial; even so, its importance as a tool for understanding ecological complexity cannot be understated. Although the details of this controversy have been explored elsewhere (Peck 2004), it is important to note that the challenges of using simulation grow out of the problems associated with equation-based models that describe processes from the top-down and miss key aspects of these systems - it is the complexity itself that we desire to explain and understand. Modeling approaches must not only represent, but must do so vis-à-vis this complexity. For example, in the study of pesticide resistance in large agro-ecosystems, where genetic, ecological, human management, and insect behavior all come together, the strategy has been to combine equations, structure, and agential behavior in ways that can only be described as a creative act (Peck 2008).

\section{Instantiations as Representations}

Representation is a well-discussed topic in philosophy of science and gets at the heart of any model of a system, including everything from paper and stick models of chemical systems to advanced mathematical models that represent the dynamics of complex physical behavior. Although van Fraassen (2008) notes that "representation is not to be subjected to definition: it is inexhaustible as a subject," he argues that a representation is a cultural artifact with a selective resemblance to its target system. Thus, models are used in a context of making representations that reveals the structure and processes of observable phenomena. The circularity in this is intentional: representations, he argues, are those things used as such.

Representations can be pictures, equations, and other traditional ways of creating resemblance relations. Physical models also can be used to construct an instance of the target system that is smaller in scale, but for which the same nomological relations are thought to hold. For example, wind tunnel experiments on airplane designs often explore the effect of smaller and more manipulable versions of airplanes to comprehend different aspects of turbulence. This procedure creates not just a symbolic representation of the target, but an instantiation. While this term is not commonly used in the literature, I think it provides a useful distinction when thinking about models and model systems, and suggests interesting applications.

Consider a particular ecological instantiation. Schenck Memorial Forest, near Raleigh North Carolina, is a 101 hectare research study site maintained by the Department of Forestry and Environmental Resources at North Carolina State University (Miller 1979). It was planted on depleted croplands among loblolly pines in 1939. This forest is now a thriving ecosystem. However, it has both artificial and 'natural' aspects of ecosystems. Because it was created by researchers, the forest can be manipulated in ways that might be unethical or otherwise problematic for more natural forests. It was built for the purpose of studying forest processes and their management, and it continues to function as such. Why not just say it is an ecosystem? That is accurate, after all. While it is an ecosystem, it is also different in that it exists to be representative of ecosystem processes for human understanding, in a way different from natural ecosystems (acknowledging all the problems with the meaning of 'natural'). It serves as a reference point because it can be manipulated and studied. Its historical features have been documented and described. It exists and is maintained for its value as representing what are thought to be spatially larger, more biotically complex, and historically less welldocumented forests. An 'experimental' forest does not seem to represent the inherent wildness of this purposefully planted forest. It is more than a controlled experiment, and less than a fully natural forest.

An instantiation is a representation and is used as such (van Fraassen 2008), but it also becomes an instance of the target system. It contains many but not all of the components of the reference system, and the components stand in reciprocal relationships that are similar to how they obtain in a natural system. On a more abstract level, trees can be treated as objects that stand in specific relationships with other objects in 
Schenck Memorial Forest. These objects have manifestations that interact in particular measurable ways (although not all aspects of objects are given or measurable), and have capacities that can be conditionally manifest, surfacing only as ecosystem processes interact through time.

Similarly, digital ecologies are built up through the creation of objects that interact in ways thought to be similar to the target system (given all the usual caveats about similarity relations). In this way, digital objects are created to represent objects in the target ecological system, but in very real respects are designed also to be an instantiation of an ecological system. Although it is true that in one case the objects are trees largely made of carbon atoms, and in the other the objects are constructed from programming code, objects qua objects interact in operationally relevant ways. There are things about objects that are relevant just because they are objects - regardless of their material origin (for an extensive discussion of objects, see Bryant 2011). We are accustomed to this kind of material substitution in object creation; for example, we create a wing out of balsawood rather than titanium to study wing performance in a wind tunnel. But sometimes it is less important what the material substance is in a model instantiation, as long as we use the objects created to interact in appropriate ways to represent features of our target system.

A digital representation has certain advantages as an instantiation of an ecological system. Complete experimental control is possible in a computer simulation in which objects are created in ways thought to behave analogously to objects in a real system. In this type of ecological instantiation, processes are uncovered and behaviors emerge because the agent-based system is an ecology itself. An ecology of digital objects, but an ecology nonetheless.

I recognize that there is an analogical leap from a planted forest to a digital ecology, but there is something about agent-based systems that capture ecological aspects and pattern generation differently from representations in a pictorial or equation-based sense. They are ecological, and constructed to interact in ways that the programmer cannot anticipate or control - objects interacting with objects. Instantiation is a fruitful way to think about the differences between these types of models and more formal mathematical representational systems, and can be seen by looking in some detail at the use of agent-based models.

\section{Agent-based Models}

In agent-based models (ABMs), also called individual-based models (Railsback and Grimm 2012), the individual participants in an ecological system are represented by digitally created agents, i.e., discrete computer objects with attributes designed to match agents and their environment in the target system. ${ }^{1}$ They have been used very successfully in social sciences to explore questions of regularity in social behavior and in the ecological sciences (Grimm and Railsback 2005). Agent-based systems are useful for understanding the complex interactions of organisms in ecological and evolutionary biology, as well as the cognitive division of labor in science (e.g., see Weisberg and Muldoon 2009). Like the complexity found in natural systems, these models allow complex patterns to "bubble-up" or emerge from lower-level interactions among agents. Digital organisms, for example, allow representation at multiple temporal and spatial scales, including the ecological, genetic, and evolutionary details of their local interactions. In addition, these types of modeling approaches - like their natural counterpart and target systems - are highly contingent on the specifics of time and place, and depend on the historical nature of the digital environment that has structured the ecosystem in and through modeling time.

This interplay of organisms and environment captures inherent complexity that is difficult to handle using standard analytic mathematical models, which rely on sets of complex equations to represent the various states of the target system. ABMs are fundamentally different from typical analytic models in that they are not necessarily equation-based, and the problems they can engender usually do not arise from the challenges created by using numerical methods to find approximate solution sets for analytic equations. There are, however, many of the familiar problems in trying to navigate between generality, precision, and realism (Weisberg 2006, 2007; Levins 2006).

Hauhs and Lange (2006) draw a useful distinction between what they refer to as algorithmic models and interactive simulations. Algorithmic models follow a kind of 'natural law' approach in which the theories and laws of the system are mapped out with formal sets of equations. Although these sets are complex, they 
progress according to state transition equations. In interactive simulations, choices are made in which new possibilities can unfold. They use the example of a chess game or a flight simulator in which new opportunities and scenarios develop in unanticipated ways when different moves are made. This is similar to what happens in agent-based ecological models. The agents can adapt, move, and change in ways that capitalize on local conditions and circumstances, which thereby moves the simulation forward to new states that are explicitly dependent on what has happened historically within the model. The local interaction of agents can generate emergent patterns that are unanticipated or programmed specifically; genuine novelty is produced in ABMs.

How does this occur? What allows these kinds of systems to unfold in new ways? These models often are built straight from code and designed to mimic the ecological system itself, in effect, creating a digital ecology that represents the natural ecological system. Much like computer game animations and manipulations, these models are world-representing in ways that are interactive and code-based. Of course, all computer modeling involves building and executing code, but in ABMs the stage of representing the target systems by formal equations is often bypassed.

ABMs produce challenges to thinking about both modeling and scientific representation. The typical $\mathrm{ABM}$ will contain computer code that defines the agent organisms and their attributes, and how these attributes affect interactions among agents within the digital ecology. Analytic models may play a role in $\mathrm{ABMs}$, such as one of the components of a complex simulation, but not necessarily, and when they do the manner of interaction is more complex. Winsberg (2010) points out that models often represent natural processes at different scales or under different circumstances using different theoretical commitments. For example, if processes operate at different scales, then different theories might be used to separately describe the two interacting scales. To solve the problem of modeling cross-scale interaction effects, different sets of equations using the theoretical commitments thought to obtain at each scale pass information between the scales in order to get a multi-scale representation of the process. ABMs differ in that the aim is for the agents themselves to create the conditions under which the multi-scale structure arises from the effects of the interactions among agents.

ABMs only represent the agent states themselves and the relationships that obtain from the interaction of the agents in their digital world. The behavior of the model emerges from these interactions - with all of the usual problems of choosing what to model, what to include or exclude, the level of abstraction, the type of idealizations, etc. In these respects, there are problems that are common to both analytic models and the simulations of ABMs. These problems are inherent in any modeling enterprise, but they can exhibit novel aspects that demand new philosophical consideration. For example, consider this description of computational modeling:

We may say that, as a matter of fact, scientists reach certain conclusions by using computational models that are (1a) usually built starting from a piece of theory (though without deducing them from theory), (1b) not deduced from data (and background knowledge, say) and (1c) constructed using a variety of different sources and techniques including approximations, idealizations and so forth (Frigg and Reiss 2009, 7).

For ABMs, much of this is not true in ecological systems. (1a) is rarely true because of the difficulty of finding general ecological theories that apply across a wide range of organisms, and the deeper suspicion that we do not have any in the same sense as models found in the physical sciences. Because we usually do know something about agential behavior through data, and it is used to construct the simulation, (1b) is not true. (1c) is true but involves such a variety of considerations in ABMs that it is unclear whether Frigg and Reiss have imagined them in what they propose here. In short, ABMs are a new and different beast. But in light of these major differences, and additional interpretive problems (see below), there is a natural question of why we would even use ABMs at all. This question has been the focus of a rather rigorous debate in ecology about whether simulation models are ever useful for making general claims about nature (for a review, see Peck 2004). They not only should be used, but they are a crucial tool for understanding ecological systems because they are inference-generating representations. 
ABMs present several new problems for understanding how models represent their target systems, and what role they can play in scientific discourse. The suggestion that simulation models present no new challenges to the philosophy of modeling may be true for typical simulations based on systems of analytic models. However, ecological simulation - in the sense of instantiations from ABMs - does seem different enough from standard modeling to pose representational problems that warrant distinct scrutiny. This is especially true when the simulation models are not derived from systems of equations, but instead built up directly from rule-based computer language coding.

\subsection{Examples from ant behavioral ecology}

Some of the challenges with representations derived from ABMs begin to appear when we examine how these models are constructed. First, the representation is constructed in a nested hierarchy of code-based structures. For example, the 'creature' may be represented as an algorithmically coded object defined by a series of variables denoting its attributes. These might include things like location, health, whether it has fed, how much energy it has remaining, sex, genetic code, or a host of other attributes thought to be relevant in representing the target agent. This is a representation more akin to creating a 'picture' of attributes relevant to the modeled agent. Second, the ecological arena must be constructed by representing the ecological environment in which the organisms act. This may be dynamic and change as the model progresses. Third, the process rules of the simulation must be specified. What happens when the agents interact? Do they mate, feed, kill, defend territory, or reproduce and create new agents? What happens is dependent on the ecology for which the representation is intended, but all ABMs must contain rules for agent interaction and environment specific behavior. Time and ordering relations must be modeled as well. An extraordinarily complex computer code is required to handle the agents and their interactions.

Examples from ant ecology can be used to illustrate these aspects of how ABMs are constructed, as well as how they differ from other simulation models (such as those used in the physical sciences), and even how they become experiments. Robinson et al. (2008) constructed a simulation model based on experiments conducted on Pharaoh's ant, Monomorium pharaonis. Figure 1A shows the state changes in the modeled antagent behavior depending on environmental context. Figure 1B shows the experimental set-up designed to capture actual ant agential interaction. While the details of these findings are important for ant ecology, I want to draw out some of the philosophical problems orbiting around how the model was built, how it was used, and what was learned from it.

The ABM is derived from a set of previous foraging experiments, literature review, and heuristic pictures that are realized in Figure 1B, but there is little theoretical guidance of the kind found in physical science modeling.

The aim of this model is to investigate how the foraging success of a colony of trail-laying ants is affected by the combined use of attractive and repellent trail pheromones, and in particular to determine the advantages of having a repellent pheromone (Robinson et al. 2008, 252).

Their goal is to reproduce agents that behave in certain ways that formally resemble the foraging ants - to construct an instantiation of the system. This differs from attempts to represent or depict using a mathematical model. The ABM is built up from MatLab code (Robinson et al. 2008, Supplemental Information), which captures the heuristics in Figure 1. For example, ant-agent decision is coded as:

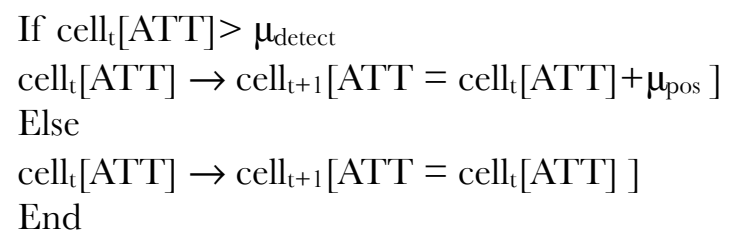


A

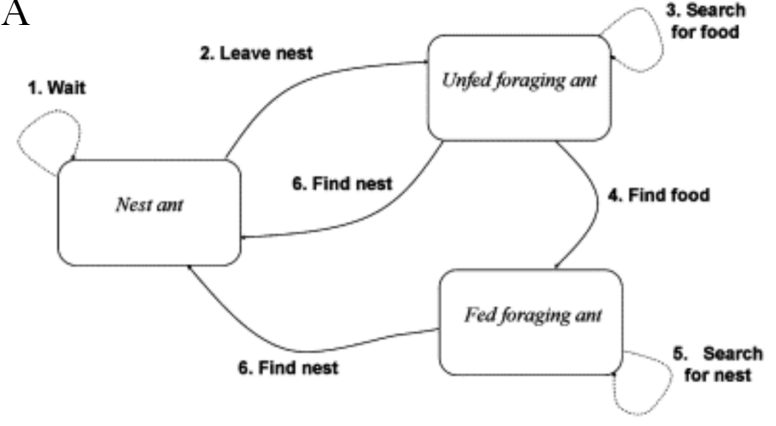

B

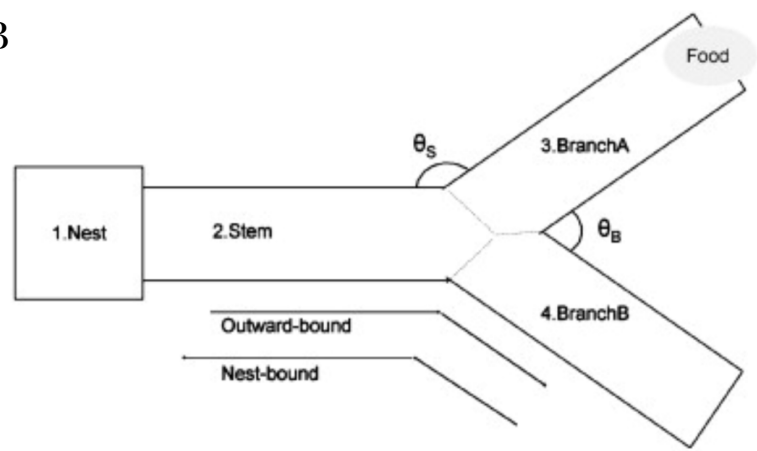

Figure 1 - A: Schematic map of ant-agent behavior found in the model described in the text (from Robinson et al. 2008, 251). In this model, ants are represented as agents that move though the different behaviors outlined in the connecting edges in this flow diagram. The ant-agents change feeding behavior state according to the nodes, illustrated by the boxes, which represent states that individual ants can enter into during a run of the model. B: Experimental set-up used as the target system for the agent-based model described in the text and used to explore ant behaviors (from Robinson et al. 2008, 251).

Pheromone decay is modeled with standard exponential decay functions in this model, but the equations are embedded in code differently than what is found when physical behavior is described with systems of analytic equations. In addition, the equations in the ABM are constructed in a more ad hoc fashion. Here the creation and running of the code is an attempt to instantiate a specific experimental situation directly, which in turn is thought to inform questions about ant foraging behavior in nature. The computer acts as a tool to investigate ants by recreating the system in a controllable way. This control means the model is easily and inexpensively manipulated. To examine ant foraging behavior in the laboratory experimentally would require vast resources; to do so in the field would be nearly impossible. The computer objects are given the individual attributes of ants and the behavioral rules that ants are thought to follow in their interactions with other ants and in their environment. Patterns observed in the ABM can be checked against real world ants and then expanded to make claims that go beyond what is manageable in the laboratory or in field studies.

The ABM also produces emergent and self-organizing behavior, which helps to explain a feature of ant foraging (repellent pheromone increases the robustness and flexibility of foraging) and offers ideas for further experiments. In the complex situation modeled here, it is unlikely that these results concerning ant behavior could be discovered apart from the agent-based approach (or at least not captured so easily). The model then becomes inference-generating in ways useful to myrmecologists in their ongoing inquiry into ant behavior.

\subsection{Do agent-based models present unique features to philosophy of science?}

ABMs allow us to instantiate a system in ways that were difficult before the computer revolution. Still, they stand, as all models do, autonomously from the world they were intended to represent:

...although the behavior of the computer models is determined only by the program and the internal logic of the computer and is thus deterministic, it still is outside the complete control of the modelers since they are not omniscient with regard to deductive consequences. Indeed, researchers that devise computer simulations often refer to their work as experiments and thereby emphasize the autonomy of their models (Schlimm 2009, 528; see also Morrison and Morgan 1999).

One place to look for new types of philosophical problems in ABMs is with respect to the generation of emergent behavior from agent interaction. "Emergence" can mean many things and tends to be a fraught concept. I am using it in the sense of Bedau (2008), who distinguishes three concepts of emergence (nominal, weak, and strong). In all three types, the foundational element of emergence is the idea that a property is emergent if it is a property that can be possessed by the macro scale but not the micro scale. A 
classic example of this is the liquidity possessed by water in a container that is not possessed by a single water molecule.

Nominal emergence obtains when wholes are dependent on their parts but also autonomous from their parts in the sense that the parts do not have the properties of the whole. Nominal emergent properties result from the parts coming together to make a whole. To take a biological example, a herd of wildebeest is a herd just as a result of individual wildebeest aggregating in a certain way. The herd emerges because of individual behavior, but can have advantages like many eyes watching for predators.

Strong emergence obtains when properties of a system exhibit "supervenient powers that create irreducible causal powers" (Bedau 2008, 158). Although these properties are dependent on the micro-level properties, they manifest causal powers that cannot be reduced. These are sometimes thought to be extra-scientific in that they cannot - in principle - be explored through reductive methods. The subjective experience of the mind is sometimes claimed as an example but there is skepticism about whether strong emergence is a coherent concept.

Bedau defines weak emergence as: "The system's global behavior derives just from the operation of microlevel processes, but the micro-level interactions are interwoven in such a complicated network that the global behavior has no simple explanation" (Bedau 2008, 160). More specifically, he argues that weak emergence is not derivable except through simulation; micro-level state changes are a function of the situation of interactions that obtains for micro-level states. A clear example would be a beehive in which individual bees are making 'decisions' based upon the needs of the hive and the situation within the hive. A bee can determine the conditions and needs of a hive and then change its behavior accordingly. Within a beehive, individual bees are making decisions, but they are exchanging information with each other, assessing the situation within the hive, and evaluating the outside world in complex ways that make the hive much more than an aggregate of bees in the same sense that a herd is just an aggregate of wildebeest. Although this is a continuum, and there is some assessment/evaluation going on within a herd (what is my neighbor looking at and why is it so nervous?), the assessment and behavior are not as complex as the hive, nor is information used to create such emergent properties.

Bedau argues that emergent systems may be recognized only through simulation. Questions about representing the back-and-forth between emergent systems and their simulation have not received much attention outside of the field of generating emergent behavior in simplistic cellular automata models. Philosophical discourse on emergence, however, is in its infancy, especially in modeling ecological systems. There is still wide disagreement on how to define emergent behavior, and discussions of how to model it are equally problematic. However, given the complexity of natural systems, it is likely that we will need multiple perspectives on ABMs to even approach these problems (Mitchell 2002; Mitchell and Dietrich 2006). Wimsatt (2007) views false models as components of theories, and Levins pointed out that true theories become manifest in the intersection of 'independent lies' (Levins 1966). This idea needs careful attention in the use of ABMs because of the different ways these models work as pragmatic, explanatory, and predictive devices for complex ecological systems (Odenbaugh 2005a). Their use requires at least some new interpretive frameworks. For example, Railsback and Grimm (2012) encourage us to focus on patterns that arise from agent-based simulation. Focusing on these patterns brings into view new ways of analyzing ABMs compared to the statistical analyses used in more traditional modeling methods. It also may require the development of new methods based on pattern recognition techniques, which suggests further philosophical considerations as well.

\section{Agent-based Models as Fictions}

Additional philosophical problems arise when trying to understand exactly what these types of models are. Godfrey-Smith (2009) views models as narratives; models are constructed as structures with characters and events that unfold in time. They are fictions capturing truths about the world in forms that both represent in the traditional sense, but also contain truths that are true within the model. In other words, there are truths in narratives that can be taken as true within the given system. (For example, the truth that Alice and the rabbit, in the book Alice in Wonderland, are both mammals even though this is not stated.) When 
the model is just considered as a description of a world, and bracketed from its real-world target, there are truths about the fictive world described by the model. From this view, ABMs introduce protagonists into a narrative, with interesting implications.

Consider again the ABMs for ant behavior. Both models have their origin in narratives and arise as an act of narrative discourse. When I have built these kinds of complex ecological models, I have worked with biologists and others who might use them. We discussed verbally what should go into the model, and what should be left out. We reflected on the scientific literature and then articulated stories about how we thought the ecologies behaved. We drew on many others, both from the literature and in conversation, whose deep acquaintance with the ecology of these systems, and the processes that structure the interactions in the natural world, inform our current views. It was not until after we had worked out a detailed narrative structure that attempts at formalism were made and our English-based discussions and descriptions were translated directly into code. The narrative aspects structured how we thought things unfolded, what trajectories were ecologically relevant, and how to incorporate probabilities and their distributions; possibilities based on how things proceed locally and historically were first hashed out in discussion. Then the narrative was coded in a computer programming language directly from these 'stories.' (The researchers who built the ant model proceeded similarly.) Even though the complexity of the final model was beyond our ability to cognitively handle, narrative was still an embedded part of what was accomplished. The computer was then able to combine these fictions into a coherent story, from which we could read off the results, but in ways we could not have anticipated.

This may hold for all models, but it is especially true for ABMs in which the translation from scientific narration (including field studies, data, laboratory experiments, theory, intuition, induction, deduction, and abduction) into code occurs in a single step with minimal stops at formalism, such as differential equations and other analytic models. The agential properties and how the agent's interact is where the representations are made explicit, rather than at higher levels of the system.

The approach used in ABMs carries certain strengths that are needed to represent highly complex ecological systems, which are interacting at multiple scales with multiple kinds of players within the same system. Since ongoing interaction among the players structures higher-level patterns, which then feed back into lower-level structure, capacities either can be realized or remain dormant according to historical and local conditions in the model. As a result, capacities of these systems become more manipulable, and the model can yield insights that emerge from these local interactions themselves.

Moreover, using the bottom-up approach found in digital ABMs allows one to observe the development of emergent properties much like those found in real systems, with part-whole relationships explicitly instantiated. There has been only limited philosophical engagement with what it means to represent emergence, and what problems or concerns might arise in representing this aspect of reality. Do emergent features of digital systems arise in the same way as emergent features do in real-world systems? Would we expect the patterns that emerge in simulation to represent the patterns found in real systems? Answers to these questions lead to further questions: How do we interpret representational aspects of emergence? Should it be with patterns, with experiments on the model, or with standard statistical approaches to data reduction and exploration? Likely, all of the above.

It is important to keep in mind one point that has not been explored adequately here: there are deep interpretive problems that are a consequence of these models being massively complex. Making explicit what binds these models to their real-world counterpart is a part of the problem; because of the complexity, we often lack the same epistemic purchase that we lack with real, complex ecology (see Humphreys 2009). In these types of models it becomes even more problematic because of the number of parts, the complexity of interactions among the parts, and how these systems are constructed. One of the most vexing issues in ecological simulation is how these complex representations should be interpreted, understood, and scientifically defended.

One of the most common sources of frustration that scientists express about the philosophy of science is its detachment from the often brutal realities of getting theory into contact with data and how a scientific representation is applied to a real system involves considerably more than is included in the traditional semantical concerns of reference, meaning, and truth (Humphreys 2009, 619). 
I have directly encountered these frustrations as an ecological modeler. Sugden (2011) rightly notes that models provide a discovery process that goes beyond their simple role as representations and generate structure for worlds that offers 'explanations in search of observations.' This means models provide a framework for theorizing prior to data collection. But at some point our models must confront the world. One way to bring these sorts of models into dialogue with the real-world is through a hermeneutical approach (Peck 2008). There are five aspects of philosophical hermeneutics that may be useful in complex ecological simulation: (1) an openness to multiple perspectives allowing for multiple levels of scientific pluralism, (2) the hermeneutic circle, a back-and-forth of active communication among both modelers and ecologists; (3) the recognition of human factors and the nature of human practices in simulation modeling, including the role of judgments and choices in the modeling enterprise; (4) the importance of play in modeling; and, (5) the non-closed nature of hermeneutic engagement, which implies continued dialogue and a recognition of the situatedness, incompleteness, and tentative nature of simulation models.

Frigg (2010) captures this sentiment succinctly when he describes simulations as authorized games of make-believe. There is something right in suggesting that simulations are about performing an authorized game. Nevertheless, this has to be combined with the idea that a model is used in a back-and-forth movement between real-world data and the model itself. This movement is one in which the model informs the on-the-ground science of given ecologies by providing such things as hypotheses to test, explanatory indications to investigate, and predictions to confirm. However, the same things must be brought back to the modeling efforts to refine, test, and confirm the model. Only in this back-and-forth movement can the complexity of these types of models be tamed.

\section{Conclusions}

In summary, at least three philosophical problems arise in ABMs:

(I) Emergence and complex systems. What does it mean to represent an emergent system in the formal sense? What problems arise in the process of creating the representation? Why should emergence in a model relate to real-world emergence? Emergent systems likely will help us explain unresolved questions in evolutionary ecology, such as the apparent increase of organismal complexity over time (Bedau 2009) and the role that niche construction may play in this process (Korb and Dorin 2011). There is widespread agreement that simulation models may be the only way to sort out these kinds of emergent properties, and there is a strong need to tease out how emergence can be studied with ABMs (and other models).

(II) Mapping complexity relations between model and world. Representing complex systems with ABMs raises new problems about how these models are brought into a relationship with the real-world vis-à-vis complexity. Addressing the novel problems raised by ABMs may require hermeneutical approaches to tease out these representational relationships. Part of the reason for this is the way that models are translated directly from narrative structures into code without intervening stops in analytic/mathematical formalism.

(III) Instantiation. In many ways, ABMs are a representation-type of the ecological or evolutionary system, and become an instantiation or exemplar of the class of systems targeted. Real objects, holding similar partwhole relationships to their counterparts in the target system, interact together in ways thought to be relevant to understanding such systems. Although not altogether divorced from representation, instantiation does different work than representation in that it is a dynamic exemplar of the same sort of system.

\section{Literature cited}

Bedau, M.A. 2008. Downward causation and autonomy in weak emergence. In: Emergence: Contemporary Readings in Philosophy and Science. Ed. by M.A. Bedau and P. Humphreys. Cambridge, MA: MIT Press. 
Bedau, M.A.. 2009. The evolution of complexity. In: Mapping the Future of Biology. Ed. by A. Barberousse, M. Morange and T. Pradeu, Berlin: Springer.

Bryant, L.R. 2011. The Democracy of Objects. Ann Arbor, MI: Open Humanities Press

Caprio, M.A., N. Storer, M.S. Sisterson, S.L. Peck, and A. de H.N. Maia. 2008. Assessing the risk of the evolution of resistance to pesticides using spatially complex simulation models. In: Global Pesticide Resistance in Arthropods. Ed. by M.E. Mota-Sanchez, D. Whalon, R.M. Hollingworth. Cambridge, MA: CABI Publishing.

Colyvan, M., S. Linquist, W. Grey, P. Griffiths, J. Odenbaugh, and H.P. Possingham. 2009. Philosophical issues in ecology: recent trends and future directions. Ecology and Society 14:22.

Cooper, G.J. 2003. The Science of the Struggle for Existence: On the Foundations of Ecology. Cambridge: Cambridge University Press.

De Roos, A.M. and L. Persson. 2005. Unstructured population models: Do population-level assumptions yield general theory? In: Ecological Paradigms Lost: Routes of Theory Change. Ed. by K. Cuddington and B. Beisner. Burlington, MA: Elsevier Academic Press.

Frigg, R. 2010. Models and fiction. Synthese 172: 251-268.

Frigg, R. and J. Reiss. 2009. The philosophy of simulation: hot new issues or same old stew? Synthese 169: 593-613.

Godfrey-Smith, P. 2009. Models and fictions in science. Philosophical Studies 143:101-116.

Grimm, V., and S.F. Railsback. 2005. Individual-based Modeling and Ecology. Princeton: Princeton University Press.

Grimm, V., E. Revilla, U. Berger, F. Jeltsch, W.M. Mooij, S.F. Railsback, H-H. Thulke, J. Weiner, T. Wiegand, and D.L. DeAngelis. 2005. Pattern-oriented modeling of agent-based complex systems: Lessons from ecology. Science 310: 987-991.

Hall, C.A.S. 1988. An assessment of several of the historically most influential theoretical models used in ecology and the data provided in their support. Ecological Modelling 43: 5-31.

Hauhs, M. and H. Lange. 2006. Foundations for the simulation of ecosystems. In: Simulation: Pragmatic Construction of Reality. Ed. by J. Lenhard, G. Küppers, and T. Shinn, Dordrecht: Springer.

Humphreys, P. 2002. Computational models. Philosophy of Science 69: S1-S11.

Humphreys, P. 2009. The philosophical novelty of computer simulation methods. Synthese 169: 615-626.

Korb, K. and A. Dorin. 2011. Evolution unbound: releasing the arrow of complexity. Biology \& Philosophy 26: 317-338.

Levins, R. 1966. Some demographic and genetic consequences of environmental heterogeneity for biological control. Bulletin of the Entomological Society of America 15: 237-240.

Levins, R. 2006. Strategies of abstraction. Biology \& Philosophy 21: 741-755.

Miller, W.D. 1979. A History School of Forest Resources 1929-1979. North Carolina State University, Department of Forestry and Environmental Resources: http://cnr.ncsu.edu/fer/dept/depthis.html.

Mitchell, S.D. 2002. Integrative pluralism. Biology \& Philosophy 17: 55-70.

Mitchell, S.D. and M.R. Dietrich. 2006. Integration without unification: An argument for pluralism in the biological sciences. The American Naturalist 168:S73-S79.

Morrison, M. and M.S. Morgan. 1999. Models as mediating instruments. In: Models as Mediators. Ed. by M.S. Morgan and M. Morrison. Cambridge, UK: Cambridge University Press.

Odenbaugh, J. 2005a. Idealized, inaccurate but successful: A pragmatic approach to evaluating models in theoretical ecology. Biology \& Philosophy 20: 231-255.

Odenbaugh, J. 2005b. The "structure" of population ecology: Philosophical reflections on unstructured and structured models. In: Ecological Paradigms Lost: Routes of Theory Change. Ed. by K. Cuddington and B. Beisner. Burlington, MA: Elsevier Academic Press.

Peck, S.L. 2004. Simulation as experiment: a philosophical reassessment for biological modeling. Trends in Ecology and Evolution 19: 530-534.

Peck, S.L. 2008. The hermeneutics of ecological simulation. Biology \& Philosophy 23: 383-402.

Railsback, S.F., and V. Grimm. 2012. Agent-Based and Individual-Based Modeling. Princeton: Princeton University Press. 
Robinson, E.J.H., F.L.W. Ratnieks, and M. Holcombe. 2008. An agent-based model to investigate the roles of attractive and repellent pheromones in ant decision making during foraging. Journal of Theoretical Biology 255: 250-258.

Roughgarden, J. 2009. Is there a general theory of community ecology? Biology \& Philosophy 24: 521-529.

Schlimm, D. 2009. Learning from the existence of models: On psychic machines, tortoises, and computer simulations. Synthese 169: 521-538.

Sugden, R.. 2011. Explanations in search of observations. Biology \& Philosophy 26: 717-736.

van Fraassen, B.C. 2008. Scientific Representation: Paradoxes of Perspective. Oxford: Clarendon Press.

Vellend, M. 2010. Conceptual synthesis in community ecology. The Quarterly Review of Biology 85:183-206.

Weisberg, M. 2006. Forty years of 'The Strategy': Levins on model building and idealization. Biology \& Philosophy 21: 623-645.

Weisberg, M. 2007. Three kinds of idealization. Journal of Philosophy 104: 639-659.

Weisberg, M. and R. Muldoon. 2009. Epistemic landscapes and the division of cognitive labor. Philosophy of Science 76: 225-252.

Wimsatt, W.G. 2007. False models as means to truer theories. In: Re-Engineering Philosophy for Limited Beings: Piecewise Approximations to Reality. Cambridge, MA: Harvard University Press.

Winsberg, E. 2009. Computer simulation and the philosophy of science. Philosophy Compass 4: 835-845.

Winsberg, E. 2010. Science in the Age of Computer Simulation. Chicago: University of Chicago Press.

\section{Notes}

1. The word 'agent' carries baggage as something that is an agent in the sense of intentional behavior. In the context of this paper, I follow Railsback and Grimm and make no distinction between individual models and $\mathrm{ABMs}$, and 'agent-based' is expanded to include its common use in modeling as a discrete object that carries attributes that inform its interaction with other objects and its environment. For example, grains of sand used to model a sorties heap might be considered model agents of ABMs in this sense although they do not make choices.

\section{AGKNOWLEDGMENTS}

I would like to thank Stephen Downes, Anya Plutynski, and Eric Winsberg for reading earlier drafts of this paper and providing helpful suggestions and insights. I would also like to thank the participants in the MS4 Simulation Conference in Toronto, CA for the insights and discussions that helped improve this paper significantly.

Copyright (C) 2012 Author(s).

This is an open-access article distributed under the terms of the Creative Commons Attribution-NonCommercial-NoDerivs license, which permits anyone to download, copy, distribute, or display the full text without asking for permission, provided that the creator(s) are given full credit, no derivative works are created, and the work is not used for commercial purposes.

ISSN 1949-0739 